\title{
Energy Efficiency Optimization for D2D Communications Underlaying UAV-assisted Industrial IoT Networks with SWIPT
}

\author{
Zhijie Su, Wanmei Feng, Student Member, IEEE, Jie Tang, Senior Member, IEEE, Zhen Chen, Member, IEEE, \\ Yuli Fu, Nan Zhao, Senior Member, IEEE, Kai-Kit Wong, Fellow, IEEE
}

\begin{abstract}
The industrial Internet of Things (IIoT) has been viewed as a typical application for the fifth generation (5G) mobile networks. This paper investigates the energy efficiency (EE) optimization problem for the device-to-device (D2D) communications underlaying unmanned aerial vehicles (UAVs)-assisted IIoT networks with simultaneous wireless information and power transfer (SWIPT). We aim to maximize the EE of the system while satisfying the constraints of transmission rate and transmission power budget. However, the designed EE optimization problem is non-convex involving joint optimization of the UAV's location, beam pattern, power control and time scheduling, which is difficult to tackle directly. To solve this problem, we present a joint UAV location and resource allocation algorithm to decouple the original problem into several sub-problems and solve them sequentially. Specifically, we first apply the Dinkelbach method to transform the fraction problem to a subtractive-form one, and propose a mulitiobjective evolutionary algorithm based on decomposition (MOEA/D) based algorithm to optimize the beam pattern. We then optimize UAV's location and power control using the successive convex optimization techniques. Finally, after solving the above variables, the original problem can be transformed into a single-variable problem with respect to the charging time, which is linear and can be tackled directly. Numerical results verify that significant EE gain can be obtained by our proposed algorithm as compared to the benchmark schemes.
\end{abstract}

Index Terms-Energy efficiency (EE), device-to-device (D2D) communications, unmanned aerial vehicle (UAV), resource allocation.

This work has been supported in part by the National Natural Science Foundation of China under Grant 61971194, in part by National Key Research and Development Project under Grant 2019YFB1804100, in part by Key Research and Development Project of Guangdong Province under Grant 2019B010156003, in part by the Natural Science Foundation of Guangdong Province under Grant 2019A1515011607, in part by the Fundamental Research Funds for the Central Universities under Grant 2019JQ08, and in part by the Research Fund Program of Guangdong Key Laboratory of Aerospace Communication and Networking Technology under Grant 2018 B030322004. (Corresponding author: Jie Tang.)

Z. Su, W. Feng, J. Tang, Z. Chen, and Y. Fu are with the School of Electronic and Information Engineering, South China University of Technology, Guangzhou 510641, China (email: 201921011697@mail.scut.edu.cn; eewmfeng@mail.scut.edu.cn; eejtang@scut.edu.cn; chenz@scut.edu.cn; fuyuli@scut.edu.cn).

N. Zhao is with the Peng Cheng Laboratory, Shenzhen 518066, China, and also with the School of Information and Communication Engineering, Dalian University of Technology, Dalian 116024, China (e-mail: zhaonan@dlut.edu.cn).

$\mathrm{K}$. Wong is with the Department of Electronic and Electrical Engineering, University College London, London WC1E 6BT, U.K. (e-mail: kaikit.wong@ucl.ac.uk).

\section{INTRODUCTION}

Industrial Internet of Things (IIoT), as a subset of IoT devices for industrial applications, brings together a plenty of smart devices to collect and process massive amounts of industrial data. Massive Machine-Type communications (mMTC), which is an important scenario in the fifth generation (5G) mobile networks, is capable of supporting massive connections of IIoT devices [1]. Nevertheless, a massive number of connected IIoT devices will cause the explosive growth of data traffic in IIoT networks, resulting in enormous power consumption [2]. Thus, how to improve the energy efficiency (EE) of IIoT network is still an open problem.

Simultaneous wireless information and power transfer (SWIPT), which can realize the simultaneous transmission of wireless information and energy so as to extend batterylife of IIoT nodes, has attract great attention recently [3]. In addition, Device-to-Device (D2D) communication is also one of the key technologies in 5G mobile networks. D2D network allows direct communication between the devices, which can enhance the spectrum utilization and mitigate the traffic load on the base stations (BSs). In recent years, many researchers have focused on investigating the resource allocation problems in D2D communication [4]-[8]. In [4], the authors focused on the power allocation in D2D networks, and confirmed that the combining SWIPT and D2D could further improve the EE. In [5], the authors considered the D2D communication underlaying cellular networks, and developed a resource allocation algorithm to maximize the throughput of the D2D systems. In [6], the authors considered the D2D underlaid cellular networks, and proposed a mode selection algorithm to maximize the sum rate of the devices. In [7], the authors presented a distributed coalition formation algorithm to maximize the EE of D2D multimedia system. In [8], the centralized and distributed D2D communication scheduling methods were designed to maximize the throughput of the cellular-aided D2D networks.

However, when IIoT devices are deployed in remote areas or disaster areas, it is inefficient to establish communication links with traditional base stations (BSs) due to long-distance transmission. Owning to the advantages of great maneuverability, wide coverage, and high flexibility, unmanned aerial vehicles (UAVs) have been widely deployed in various scenarios to provide wireless services for users especially for the edge users. Therefore, UAVs can act as the air BSs to provide 
efficient information and energy transmission services for users that distributed in geographically constrained areas. In [9], the authors focused on the UAV-based communication networks and proposed an analytical method to adjust the height of UAV to maximize the number of served users. In [10], the authors optimized the location of the UAV, in order to employ the least number of UAVs to fully cover a specific area while meeting the communication requirements. In [11], the authors investigated the uplink transmission in UAV-assisted wireless networks, where an optimal location planning method was developed to optimize the transmission rate of the devices. In [12], the authors further considered the UAV-assisted wireless power transfer system, and maximized the sum received power of all devices by the successive convex programming-based trajectory design method. In [13], a sum rate maximization problem was investigated in a UAVaided mmWave network, where the 3D deployment of UAV, power allocation and beam pattern were considered. In [14], a secrecy rate optimization problem was studied in a secure UAV-assisted SWIPT network, and solved by an alternative optimization method.

In addition, non-orthogonal multiple access (NOMA) is viewed as a key technique to achieve high spectrum efficiency in the next generation wireless communication systems. In particular, by exploiting superposition coding (SC) at the transmitters and successive interference cancellation (SIC) at the receivers, NOMA schemes enable multiple users served at the same resource block. Moreover, the users with great channel condition are assigned a lower transmit power, while the users with poor channel condition are allocated a higher transmit power. These features make NOMA capable of guaranteeing the fairness of users as well as improving the spectral efficiency [15], [16]. For example, the authors in [15] developed a dynamic power allocation algorithm to minimize the outage probability and maximize the average rate of the downlink and uplink NOMA systems. In [16], an iterative algorithm was developed to maximize the EE in a NOMA communication network with SWIPT, where the power allocation was taken into account. Simulation results demonstrated that the combination of SWIPT and NOMA could further improve the EE of the communication network. Based on the prior works on NOMA-enabled wireless networks, the applications of UAVs within NOMA networks can further improve the system throughput since they can shorten the transmission distance and increase the channel gain [17]-[19]. In [17], the authors investigated the time sharing (TS) NOMA networks, and developed a resource allocation algorithm to maximize the EE of the UAV-TS-NOMA networks. Besides, the energy consumption minimization problem for the NOMAaided UAV data collection system was investigated in [18]. To guarantee the quality of service (QoS), the authors developed a data collection optimization algorithm using the generalized benders decomposition methods. The authors in [19] studied the UAV-assisted NOMA networks for emergency communications. Firstly, a deep-Q-learning-based scheme was proposed to maximize the sum rate of the UAV-enabled uplink NOMA networks. Then, for the multi-UAV enabled NOMA networks, a joint UAV deployment and resource allocation algorithm was provided to maximize the minimum throughput of the devices.

In fact, although the sum-throughput of the IIoT networks can be improved by the NOMA based UAV communication systems, the performance gain is very limited. This is because the received signal strength is significantly degraded by severe path loss, especially for IIoT devices distributed in wide areas. In this case, UAVs require a higher power consumption to satisfy the QoS requirements, which in turn decreases the EE performance. To tackle this problem, beamforming technique is used to focus the signal strength toward the receivers for improving the system performance [20]-[23]. The authors in [20] considered the massive multiple-input multiple-output (MIMO) hybrid beamforming system, and proposed a beamoriented digital predistortion technique to obtain linearization of the transmitted signal. Furthermore, the authors in [21] aimed at maximizing the total energy harvest of the users by jointly optimizing the placement of UAV, the beam pattern and the charging time in a UAV-enabled wireless energy transfer system. The authors in [22] provided a low complexity resource assignment algorithm to maximize the $\mathrm{EE}$ for a wireless powered sensor system, and applied the beamforming technique to further improve the energy transfer efficiency. The work in [23] aimed to maximize the minimum EE of the users for the multicell multiuser joint transmission systems, and a fairness energy efficient algorithm was provided to adjust the beamforming vector.

\section{A. Contributions}

Previous works focus on studying the resource allocation problem for the D2D communication networks [6], [7], and UAV-assisted D2D communication networks [24], [25], where the throughput is maximized through the alternative optimization methods. However, these frameworks cannot be directly used to provide simultaneous information and energy flow for the energy-constrained IIoT devices. In addition, the works in [9]-[11] study the resource allocation schemes in UAV-enabled communication networks, which cannot be directly employed to deliver effective services for devices deployed in remote areas, since only a single antenna is considered. The works in [14], [26] focus on investigating the effective algorithms to maximize the received power in UAV-assisted SWIPT networks. These works cannot be directly applied to enhance the $\mathrm{EE}$ of the system. Motivated by the aforementioned observations, we investigate the EE maximization problem in a UAVassisted D2D communication IIoT network. In addition, the beanforming technique and NOMA are considered to further enhance the system performance. The main contributions of this paper are summarized as follows:

- We formulate the design of resource allocation for IIoT devices in a UAV-assisted D2D communication network with SWIPT. We aim to maximize the EE at all IIoT devices while satisfying the constraints of devices' QoS demands and maximum transmit power. The considered optimization problem is non-convex involving joint optimization of the UAV location, power allocation, power splitting ratio and beam pattern, which is quite difficult to tackle directly. To tackle this problem, we develop a 
joint UAV location and resource algorithm that optimizes the variables sequentially.

- First, to tackle the non-convex optimization problem, we apply the Dinkelbach method to convert the original fractional optimization problem to a subtractive-form one. Then, we apply the successive convex optimization techniques to obtain the approximate convex optimization problems to obtain the location of UAV. After that, we adopt the multi-objective evolutionary algorithm based on decomposition (MOEA/D) [27] to control the beam pattern. In addition, we prove that the optimization problem is concave with respect to the power splitting ratio and transmit power in SWIPT phase and solve it using the standard convex optimization approaches. Besides, we also apply the successive convex optimization techniques to optimize the transmit power in D2D phase. Finally, with the solved variables, the considered problem is transformed into a linear programming problem which can be tackled by the standard convex optimization techniques.

- Numerical results verify that significant EE performance gain can be obtained through our proposed methods as compared to the benchmark schemes, thereby demonstrating the advantages of integrating NOMA and beamforming technique in UAV-assisted IIoT networks with SWIPT.

\section{B. Organization and Notation}

The remaining of this paper is organized as follows. The system model and the corresponding EE optimization problem are discussed in Section II. In Section III, we propose the iterative algorithm via jointly optimizing the location of UAV, beam pattern, power allocation, power splitting (PS) ratio and time scheduling. The numerical results are discussed in Section IV to verify the theoretical findings. Finally, conclusions are presented in Section V.

The following notations are used throughout this paper. Scalars and vectors are indicated by non-bold and bold case letters respectively. For a vector $a, a^{T}$ denotes its transpose, $a^{H}$ indicates its complex conjugate transpose, and $\|a\|$ represents its Euclidean norm.

\section{SYSTEM MODEL AND PROBLEM FORMULATION}

\section{A. System Model}

We consider a D2D communications in UAV-assisted IIoT network with SWIPT, which is shown in Fig. 1. The UAV is mounted with $M \times N$ antenna array, and $K \geq 2$ D2D pairs are equipped with one single antenna due to the limitations of the hardware size and battery power. Each D2D transmitter (D2DTX) $k=\{1,2, \ldots, K\}$ has a fixed location on the ground which is expressed as $z_{k}^{T x}=\left(x_{k}^{T x}, y_{k}^{T x}\right)$, and the $k$ th $\mathrm{D} 2 \mathrm{D}$ receiver (D2D-RX) is expressed as $z_{k}^{R x}=\left(x_{k}^{R x}, y_{k}^{R x}\right)$. The horizontal location of UAV is denoted as $z_{u}=\left(x_{u}, y_{u}\right)$, and the UAV is set to work at a fixed altitude $H$. The whole period $T_{o}$ contains two phases. In the SWIPT phase with duration $\tau_{S} T_{o}$ $\left(0 \leq \tau_{S} \leq 1\right)$, the UAV transmits information and power to

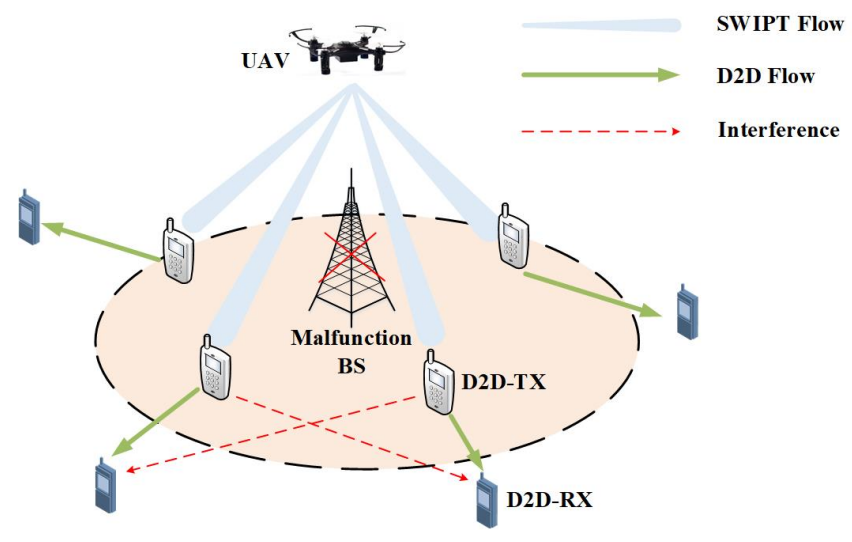

Fig. 1. Illustration of a UAV-assisted D2D communication network with SWIPT.

D2D-TXs. In the D2D phase with duration $\tau_{D} T_{o}\left(\tau_{D}+\tau_{S} \leq\right.$ 1), the D2D-TXs transmit information to D2D-RXs using the harvested energy in the SWIPT phase. For simplicity, we set $T_{o}=1$. Sepcifically, in the SWIPT phase, UAV serves as a flying BS to transmit power and information to D2D-TX using NOMA. Since the UAV base station has the advantage of mobile flexibility, we suppose that the communication links between the UAV and the D2D-TXs are line-of-sight (LOS) dominated [28]. Thus, the channel gain between the UAV and the $k$ th D2D-TX is expressed as [29]

$$
\mathbf{h}_{k}=\sqrt{\rho_{0} d_{k}^{-2}} \mathbf{a}(\theta, \phi),
$$

where $\rho_{0}$ denotes the channel power gain at a reference distance of $d_{0}=1 \mathrm{~m}$. The distance between the UAV and $k$ th D2D-TX is $d_{k}=\sqrt{\left(x_{k}^{T x}-x_{u}\right)^{2}+\left(y_{k}^{T x}-y_{u}\right)^{2}+H^{2}}$, and $\mathbf{a}(\theta, \phi)$ denotes the steering vector, which is given by

$$
\begin{aligned}
\mathbf{a}(\theta, \phi)= & {\left[1, \cdots, e^{j 2 \pi / \lambda d \sin (\theta)[(j-1) \sin (\phi)+(i-1) \cos (\phi)]},\right.} \\
& \left.\cdots, e^{j 2 \pi / \lambda d \sin (\theta)[(N-1) \sin (\phi)+(M-1) \cos (\phi)]}\right]^{T},
\end{aligned}
$$

where $\theta$ is the elevation angle and $\phi$ indicates the azimuth angle of the LOS path. $\lambda$ represents the wavelength and $d$ is the spacing between antenna elements. $i$ and $j$ denote the coordinate of antenna elements. The channel power gain from the UAV to the $k$ th D2D-TX is formulated as

$$
\left|\mathbf{h}_{k}^{H} \mathbf{w}\right|^{2}=\frac{\rho_{0}\left|\mathbf{a}^{H}(\theta, \phi) \mathbf{w}\right|^{2}}{\left(x_{k}^{T x}-x_{u}\right)^{2}+\left(y_{k}^{T x}-y_{u}\right)^{2}+H^{2}},
$$

where $\mathbf{w}$ denotes the beamforming vector. $\mathbf{E}(\theta, \phi)=$ $\mathbf{a}^{H}(\theta, \phi) \mathbf{w}$ is the synthesized pattern of the antenna array. Each D2D-TX comprises of an information decoding (ID) circuit and an energy harvesting (EH) rectification circuit. Power splitting method is adopted to split the signal into two parts, one of which is exploited for energy harvesting whilst the other is used to decode the information. The transmission power of UAV is limited to $P_{\max }$, and the power allocated to the $k$ th D2D-TX is assumed to be $P_{k}^{S}$. The PS ratio is divided into two part, where $\alpha_{k}^{S}$ is the fraction of transmission power allocated to the $k$ th D2D-TX for ID, and $1-\alpha_{k}^{S}$ represents 
the ratio for $\mathrm{EH}$. Thus, the signal received by the $k$ th D2D-TX for ID is expressed as

$$
y_{k}^{I D}=\sqrt{\alpha_{k}^{S}} g_{k}^{S} \sum_{i=1}^{K} \sqrt{P_{i}^{S}} s_{i}+N_{0},
$$

where $g_{k}^{S}=\left|\mathbf{h}_{k}^{H} \mathbf{w}\right|^{2}, s_{i}$ denotes the signal from UAV to the $i$ th D2D-TX, and $N_{0}$ is the additive Gaussian white noise (AWGN) with power $\sigma^{2}$. With successive interference cancellation (SIC) operation, the $k$ th D2D-TX will detect the $j$ th D2D-TX's information, $j<k$, and remove the information from its observation. The message for $j$ th D2DTX, $j>k$, will be treated as noise at the $k$ th D2D-TX. Thus, after applying the NOMA technique with SIC, the achievable transmission rate for D2D-TX $k$ is given by

$$
R_{k}^{S}=\log _{2}\left(1+\frac{\alpha_{k}^{S} g_{k}^{S} P_{k}^{S}}{\sigma^{2}+\alpha_{k}^{S} g_{k}^{S} \sum_{i=k+1}^{K} P_{i}^{S}}\right) .
$$

The signal received by the $k$ th D2D-TX for EH is expressed as

$$
y_{k}^{E H}=\sqrt{1-\alpha_{k}^{S}} g_{k}^{S} \sum_{i=1}^{K} \sqrt{P_{i}^{S}} s_{i}+N_{0},
$$

Then, the corresponding harvested energy at the $k$ th D2D-TX is expressed as

$$
E_{k}^{S}=\tau_{S}\left(1-\alpha_{k}^{S}\right) \eta g_{k}^{S} \sum_{i=1}^{K} P_{i}^{S},
$$

where $\eta$ indicates the energy conversion efficiency. Thus, the total energy consumption in the SWIPT phase is expressed as

$$
E_{\text {total }}^{S}=\tau_{S}\left(\zeta \sum_{k=1}^{K} P_{k}^{S}+P_{C}^{S}+P_{\text {hov }}\right)-\sum_{k=1}^{K} E_{k}^{S},
$$

where $\zeta$ represents the drain efficiency of the power amplifier, $P_{C}^{S}$ is the energy consumed by the hardware in the SWIPT phase, and $P_{h o v}$ denotes the power consumed by the UAV during hovering.

In the D2D phase, we assume that the communication links between the D2D-TX and D2D-RX is also LoS dominated due to the short-distance communication of D2D links [30]. Thus, the channel power gain from the $m$ th D2D-TX to the $k$ th D2D-RX is expressed as

$$
g_{m, k}^{D}=\frac{\rho_{0}}{\left(x_{m}^{T}-x_{k}^{R x}\right)^{2}+\left(y_{m}^{T}-y_{k}^{R x}\right)^{2}} .
$$

Let $P_{k}^{D}$ be the transmission power of the $k$ th D2D-TX. Then, the achievable transmission rate of the $k$ th $\mathrm{D} 2 \mathrm{D}-\mathrm{RX}$ is given by

$$
R_{k}^{D}=\log _{2}\left(1+\frac{g_{k, k}^{D} P_{k}^{D}}{\sigma^{2}+\sum_{i=1, i \neq k}^{k} g_{i, k}^{D} P_{i}^{D}}\right) .
$$

In addition, the total energy consumption in the D2D phase is formulated as

$$
E_{\text {total }}^{D}=\sum_{k=1}^{K} E_{k}^{D}=\tau_{D}\left(\sum_{k=1}^{K} P_{k}^{D}+P_{C}^{D}\right),
$$

where $P_{C}^{D}$ denotes the energy consumed by the hardware during the D2D phase. Therefore, the EE of the considered network is given by

$$
\lambda_{E E}=\frac{T R_{\text {total }}}{E_{\text {total }}}=\frac{\tau_{S} \sum_{k=1}^{K} R_{k}^{S}+\tau_{D} \sum_{k=1}^{K} R_{k}^{D}}{E_{\text {total }}^{S}+E_{\text {total }}^{D}} .
$$

\section{B. Problem Formulation}

In this paper, we aim to maximize the EE of the considered D2D communications underlaying UAV-assisted IIoT network while satisfying the constraints of minimum transmission rate and total transmission power of UAV. Mathematically, the optimization problem is expressed as

$$
\begin{array}{cc}
\max _{E(\theta, \phi), P_{k}^{S, D}, z_{u}, \alpha_{k}^{S}, \tau_{S, D}} \lambda_{E E} \\
\text { s.t. } \quad R_{k}^{S} \geq R_{\min }^{S}, \forall k \in \mathcal{K}, \\
& R_{k}^{D} \geq R_{\min }^{D}, \forall k \in \mathcal{K}, \\
& \sum_{k=1}^{K} P_{k}^{S} \leq P_{\max }, \\
& E_{k}^{D} \leq E_{k}^{S}, \forall k \in \mathcal{K}, \\
& \tau_{S}+\tau_{D} \leq 1 \\
& 0 \leq \tau_{S}, \tau_{D} \leq 1 \\
& 0 \leq \alpha_{k}^{S} \leq 1
\end{array}
$$

Constraints (13b), (13c) indicate that the achievable rate in the SWIPT phase and the D2D phase should satisfy the minimum transmission rate constraint $R_{\min }^{S}$ and $R_{\text {min }}^{D}$ respectively to ensure the QoS of the IIoT devices. Constraint (13d) denotes that the transmission power of UAV cannot exceed the power budget $P_{\max }$. Constraint $(13 \mathrm{e})$ guarantees that the energy consumed by each D2D-TX cannot exceed its harvested energy from the UAV. Constraints (13f) and (13g) limit the time switching ratio for SWIPT phase and D2D phase, and constraint (13h) limits the power splitting ratio for ID and EH. Problem (13) is a non-convex problem due to the coupling variables, which is challenging to solve. To solve this problem, we develop an efficient resource allocation algorithm by optimizing the above variables sequentially.

\section{THE ITERATIVE JOINT UAV LOCATION AND RESOURCE ALLOCATION ALGORITHM}

In this section, we develop an iterative joint UAV location and resource allocation algorithm where the designed problem is decoupled into several problems and solved then sequentially. Specifically, since the beam pattern design requires the beam scanning angles, the UAV location should be determined first. Then, based on the acquisition of angle information, the beam pattern is obtained. Subsequently, with the fixed UAV's placement and beam pattern design, the PS ratio and power allocation in SWIPT phase are optimized. Finally, the power allocation in D2D phase is optimized to maximize the EE of the system. Therefore, by exploiting the Dinkelbach method, the original problem is converted into a subtractive-form 
one. Then, we employ the successive convex optimization technique to optimize the UAV location. Subsequently, we propose a beamforming design method to optimize the beam pattern. In addition, the standard convex optimization approach is employed to obtain the optimal PS ratio and transmit power in SWIPT phase. The power allocation in D2D phase is determined by the successive convex optimization technique. Finally, a linear programming (LP) problem with respect to time scheduling is tackled using the standard convex optimization techniques. Since the objective function of problem (13) is in fractional form, it is difficult to tackle. Thus, we apply the Dinkelbach method [31] to solve this fractional optimization problem. Specifically, we convert the fraction problem to a subtractive-form one based on the following proposition.

Proposition 1: The achievable EE $q^{*}$ can be obtained as follows

$$
\max _{E(\theta, \phi), P_{k}^{S, D}, z_{u}, \alpha_{k}^{S}, \tau_{S, D}} U_{R}-q^{*} U_{T}=U_{R}^{*}-q^{*} U_{T}^{*}=0,
$$

where

$$
\begin{gathered}
U_{R}=\tau_{S} \sum_{k=1}^{K} R_{k}^{S}+\tau_{D} \sum_{k=1}^{K} R_{D}^{S} \geq 0 \\
U_{T}=E_{\text {total }}^{S}+E_{\text {total }}^{D} \geq 0
\end{gathered}
$$

and

$$
q^{*}=\frac{U_{R}^{*}}{U_{T}^{*}} .
$$

Proof: Please refer to [31] for a proof of Proposition 1.

From [31], the equivalent subtractive form can replace the original objective function. [31] further shows that the optimal solution can be obtained according to the conditions of the equation in Proposition 1. Thus, with the given $q$, the equivalent objective function is given by

$$
\begin{aligned}
& \max _{E(\theta, \phi), P_{k}^{S, D}, z_{u}, \alpha_{k}^{S}, \tau_{S, D}} \lambda_{E E}^{\prime} \\
= & \tau_{S} \sum_{k=1}^{K} R_{k}^{S}+\tau_{D} \sum_{k=1}^{K} R_{k}^{D}-q\left(E_{\text {total }}^{S}+E_{\text {total }}^{D}\right) .
\end{aligned}
$$

Therefore, we can tackle this subtractive-form problem by optimizing the UAV location, beam pattern, power allocation and time scheduling sequentially, and update $q^{*}$ according to (17). Furthermore, the convergence of the Dinkelbach-based method has been proved in [16].

\section{A. Location Optimization}

For a given $\left(q, E(\theta, \phi), P_{k}^{S}, \alpha_{k}^{S}, P_{k}^{D}, \tau_{S}, \tau_{D}\right)$, we focus on the optimization problem for the UAV location $z_{u}$. Problem (13) is reformulated as:

$$
\begin{aligned}
\max _{z_{u}} & \lambda_{E E}^{\prime} \\
\text { s.t. } & \log _{2}\left(1+\frac{\alpha_{k}^{S} g_{k}^{S} P_{k}^{S}}{\sigma^{2}+\alpha_{k}^{S} g_{k}^{S} \sum_{i=k+1}^{K} P_{i}^{S}}\right) \geq R_{\text {min }}^{S}, \forall k \in \mathcal{K}, \\
& \tau_{S}\left(1-\alpha_{k}^{S}\right) \eta g_{k}^{S} \sum_{i=1}^{K} P_{i}^{S} \geq E_{k}^{D}, \forall k \in \mathcal{K} .
\end{aligned}
$$

Constraint (19b) is non-convex with respect to $z_{u}$. Applying the successive convex optimization approach, $R_{k}^{S}$ is first reformulated as

$$
R_{k}^{S}=\tilde{R}_{k}^{S}-\hat{R}_{k}^{S}
$$

where

$$
\begin{gathered}
\tilde{R}_{k}^{S}=\log _{2}\left(\frac{\rho_{0} \alpha_{k}^{S}|E(\theta, \phi)|^{2}}{H^{2}+\left\|z_{k}^{T x}-z_{u}\right\|^{2}} \sum_{i=1}^{K} P_{i}^{S}+\sigma^{2}\right), \\
\hat{R}_{k}^{S}=\log _{2}\left(\frac{\rho_{0} \alpha_{k}^{S}|E(\theta, \phi)|^{2}}{H^{2}+\left\|z_{k}^{T x}-z_{u}\right\|^{2}} \sum_{i=k+1}^{K} P_{i}^{S}+\sigma^{2}\right) .
\end{gathered}
$$

Noted that $\tilde{R}_{k}^{S}$ is neither concave nor convex with respect to $z_{u}$, but convex with respect to $\left\|z_{k}^{T x}-z_{u}\right\|^{2}$. We define the local point $z_{u}^{r}$ as the given location of UAV in the $r$ th iteration. Then, we can obtain the globally lower bound of (21) by applying the first order Taylor expansion [32], which can be formulated as

$$
\begin{aligned}
\tilde{R}_{k}^{S} & =\log _{2}\left(\frac{\rho_{0} \alpha_{k}^{S}|E(\theta, \phi)|^{2}}{H^{2}+\left\|z_{k}^{T x}-z u\right\|^{2}} \sum_{i=1}^{K} P_{i}^{S}+\sigma^{2}\right) \\
& \geq \sum_{i=1}^{k}-A_{k}^{r}\left(\left\|z_{k}^{T x}-z_{u}\right\|^{2}-\left\|z_{k}^{T x}-z_{u}^{r}\right\|^{2}\right) \\
& +B_{k}^{r} \triangleq \tilde{R}_{k}^{S l b},
\end{aligned}
$$

where $A_{k}^{r}$ and $B_{k}^{r}$ can be calculated as

$$
A_{k}^{r}=\frac{\frac{P_{i}^{S} \rho_{0} \alpha_{k}^{S}|E(\theta, \phi)|^{2}}{\left(H^{2}+\left\|\mathbf{z}_{k}^{T}-\mathbf{z}_{u}^{r}\right\|^{2}\right)^{2}} \log _{2}(e)}{\frac{\rho_{0} \alpha_{k}^{S}|E(\theta, \phi)|^{2}}{H^{2}+\left\|\mathbf{z}_{k}^{T}-\mathbf{z}_{u}^{r}\right\|^{2}} \sum_{l=1}^{k} P_{l}^{S}+\sigma^{2}},
$$

and

$$
B_{k}^{r}=\log _{2}\left(\frac{\rho_{0} \alpha_{k}^{S}|E(\theta, \phi)|^{2}}{H^{2}+\left\|\mathbf{z}_{k}^{T}-\mathbf{z}_{u}^{r}\right\|^{2}} \sum_{l=1}^{k} P_{l}^{S}+\sigma^{2}\right) .
$$

With (20) and (23), (19b) can be reformulated as

$$
\tilde{R}_{k}^{S l b}-\hat{R}_{k}^{S} \geq R_{\min }^{S} .
$$

However, (26) is still non-convex due to $\hat{R}_{k}^{S}$. Thus, we introduce the slack variable $\mathbf{S}=\left\{S_{k}=\left\|\mathbf{z}_{k}^{T}-\mathbf{z}_{u}\right\|^{2}, \forall k\right\}$, which should satisfy the following constraints

$$
S_{k} \leq\left\|\mathbf{z}_{k}^{T}-\mathbf{z}_{u}\right\|^{2}, \forall k .
$$

Then, $\hat{R}_{k}^{S}$ can be reformulated as

$$
\hat{R}_{k}^{S}=\log _{2}\left(\frac{\rho_{0} \alpha_{k}^{S}|E(\theta, \phi)|^{2}}{H^{2}+S_{k}} \sum_{i=k+1}^{K} P_{i}^{S}+\sigma^{2}\right) .
$$

Since $\left\|\mathbf{z}_{k}^{T}-\mathbf{z}_{u}\right\|^{2}$ is convex with respect to $z_{u}$, we have the following inequality via the first order Taylor expansion at the given point $z_{u}^{r}$

$$
\begin{aligned}
\left\|z_{k}^{T x}-z_{u}\right\|^{2} & \geq\left\|z_{k}^{T x}-z_{u}^{r}\right\|^{2} \\
& +2\left(z_{k}^{T x}-z_{u}^{r}\right)^{T}\left(z_{u}-z_{u}^{r}\right) .
\end{aligned}
$$

By substituting (29) into (27), problem (19) is rewritten as 


$$
\begin{array}{ll}
\max _{z_{u}, S} & \tau_{S}\left(\sum_{k=1}^{K} \tilde{R}_{k}^{S l b}-\hat{R}_{k}^{S}\right)+\tau_{D} \sum_{k=1}^{K} R_{k}^{D}-q E_{\text {total }} \\
\text { s.t. } & \tilde{R}_{k}^{S l b}-\log _{2}\left(\frac{\rho_{0} \alpha_{k}^{S}|E(\theta, \phi)|^{2}}{H^{2}+S_{k}} \sum_{i=k+1}^{K} P_{i}^{S}+\sigma^{2}\right) \\
& \geq R_{m i n}^{S}, \forall k \in \mathcal{K}, \\
& \tau_{S}\left(1-\alpha_{k}^{S}\right) \eta g_{k}^{S} \sum_{i=1}^{K} P_{i}^{S} \geq E_{k}^{D}, \forall k \in \mathcal{K}, \\
& S_{k} \leq\left\|z_{k}^{T x}-z_{u}^{r}\right\|^{2}+2\left(z_{k}^{T x}-z_{u}^{r}\right)^{T}\left(z_{u}-z_{u}^{r}\right) .
\end{array}
$$

Consequently, problem (30) is convex now, which can be efficiently tackled using the standard convex optimization methods [33].

\section{B. Optimal Phased-Array Pattern}

With the fixed $\left(q, z_{u}, P_{k}^{S}, \alpha_{k}^{S}, P_{k}^{D}, \tau_{S}, \tau_{D}\right)$, the optimization problem with respect to $E(\theta, \phi)$ can be expressed as:

$$
\begin{array}{cl}
\max _{E(\theta, \phi)} & \lambda_{E E}^{\prime} \\
\text { s.t. } & R_{k}^{S} \geq R_{\min }^{S}, \forall k \in \mathcal{K}, \\
& E_{k}^{D} \leq E_{k}^{S}, \forall k \in \mathcal{K} .
\end{array}
$$

From (3) and (12), the channel power gain $g_{k}^{S}$ increases with $E(\theta, \phi)$, resulting in a significantly enhancement of the EE and achievable transmission rate. Hence, problem (31) can be rewritten as

$$
\max |\mathbf{E}(\theta, \phi)|^{2} .
$$

In this work, the $M \times N$ antenna array can be partitioned into several sub-arrays, where the steerable beams formed by the sub-arrays are assumed to be independent. Hence, problem (32) can be reformulated as

$$
\max E_{k}(\theta, \phi) .
$$

To form the directional beams, we control the side-lobe level (SLL), array gain and beamwidth simultaneously through optimizing the phase of antenna element. Mathematically, the beam pattern multiobjective optimization problem (MOP) with respect to phase $z$ can be constructed as

$$
\begin{aligned}
& \min F(\boldsymbol{z})=\left(f_{1}(\boldsymbol{z}), f_{2}(\boldsymbol{z}), f_{3}(\boldsymbol{z})\right)^{T} \\
& \text { s.t. } \boldsymbol{z} \in \mathbf{R}^{M \times N},
\end{aligned}
$$

where $f_{1}(\boldsymbol{z})=S L L(\boldsymbol{z}), f_{2}(\boldsymbol{z})=\frac{1}{|\mathbf{E}(\theta, \phi)|}, f_{3}(\boldsymbol{z})=\frac{1}{\Theta_{h, e}}, \boldsymbol{z}=$ $\left[z_{1 n}, \cdots, z_{m n}, \cdots, z_{M N}\right]^{T}$ denote the phases of the $M \times N$ antenna array. $S L L(\boldsymbol{z})=20 \log \frac{\left|\mathrm{F}_{s l l}\right|}{\left|\mathrm{F}_{m l}\right|}$ denotes the side-lobe level of the antenna array, where $\mathrm{F}_{\text {sll }}$ and $\mathrm{F}_{m l}$ represent the array factor of the maximum SLL and main lobe, respectively. $\mathbf{E}(\theta, \phi)=\mathbf{a}^{H}(\theta, \phi) e^{j \boldsymbol{z}}$ represents the synthesized pattern and $\Theta_{h, e}$ denotes the elevation plane half-power beamwidth. To tackle problem (34), we apply the MOEA/D solution [27]. The steps of the algorithm can be described as follows:

- Input: Let $\left\{N_{0}, \gamma^{i}, S\right\}$ be a set of input parameters. Here, $N_{0}$ is the number of subproblems. $\gamma^{i}=\left(\gamma_{1}^{i}, \ldots, \gamma_{d}^{i}\right)^{T}$, $i=1, \ldots, N_{0}, d$ represents the weight vector of the $i$ th subproblem. $S$ denotes the number of weight vectors in each neighborhood.
- Output: EP: a non-dominated solutions set.

- Initialization: For each $i=1, \ldots, N_{0}$, we select $S$ as the closest weight vectors of $\gamma^{i}$ by calculating the Euclidean distance, and store them in $C(i)$. Then, we produce the initial solutions $z_{1}, \ldots z_{N_{0}}$ randomly, and update the Fvalues $F V_{i}=F\left(z_{i}\right)$. In addition, we initiate the bestso-far solutions $\beta=\left(\beta_{1}, \ldots, \beta_{j}, \ldots, \beta_{N_{d}}\right)^{T}$, where $\beta_{j}=$ $\min \left\{f_{j}(z), z \in \mathbf{R}^{M \times N}\right\}$, and set EP to be empty.

- Update: For each $i=1, \ldots, N_{0}$, we choose weight vectors $z_{k}, z_{l}$ from $C(i)$, and generate the new solution $x$. Then, for $j=1, \ldots, d$, if $\beta_{j}>f_{j}(x)$, it follows that $\beta_{j}=f_{j}(x)$; If $g^{t e}\left(x \mid \gamma^{j}, \beta\right) \leq g^{t e}\left(z_{j} \mid \gamma^{j}, \beta\right)$, it follows that $z_{j}=x$ and $F V_{j}=F(x)$, where $g^{t e}\left(x \mid \gamma^{j}, \beta\right)=\max _{1 \leq t \leq d}\left\{\gamma_{t}^{j}\left|f_{t}(\boldsymbol{x})-\beta_{t}\right|\right\}$ [27]. Then, we eliminate the vector dominated by $F(x)$ from EP, if no vectors dominate $F(x)$, we add it to EP.

- Stopping: The iterations have converged.

\section{Optimal PS Ratio and Power Allocation in SWIPT Phase}

We optimize $\left(P_{k}^{S}, \alpha_{k}^{S}\right)$ respectively with the fixed $\left(q, z_{u}, E(\theta, \phi), P_{k}^{D}, \tau_{S}, \tau_{D}\right)$. The optimization problem is expressed as:

$$
\begin{aligned}
\max _{P_{k}^{S}, \alpha k^{S}} & \lambda_{E E}^{\prime} \\
\text { s.t. } \quad & R_{k}^{S} \geq R_{\min }^{S}, \forall k \in \mathcal{K}, \\
& \sum_{k=1}^{K} P_{k}^{S} \leq P_{\max }, \\
& \tau_{S}\left(1-\alpha_{k}^{S}\right) \eta g_{k}^{S} \sum_{i=1}^{K} P_{i}^{S} \geq E_{k}^{D}, \forall k \in \mathcal{K} .
\end{aligned}
$$

To tackle this problem, we first put forward the proposition which demonstrates that the objective function (35a) is concave in the transmit power and the PS ratio.

Proposition 2: The objective function is strictly concave with respect to $P_{k}^{S}$ and $\alpha_{k}^{S}, \forall k \in \mathcal{K}$.

Proof: See Appendix.

Note that the constraint (35b) can be converted as the equivalent form

$$
\sigma^{2}+\alpha_{k}^{S} g_{k}^{S} \sum_{i=k}^{K} P_{i}^{S}-2^{R_{\text {min }}^{S}}\left(\sigma^{2}+\alpha_{k}^{S} g_{k}^{S} \sum_{i=k+1}^{K} P_{i}^{S}\right) \geq 0 .
$$

Since constraint (36) is clearly linear, the optimization problem (35) is convex with respect to $P^{S}$ and $\alpha^{S}$, and can be tackled by the standard convex optimization approaches [33].

\section{Power Allocation in D2D Phase}

With the fixed $\left(q, z_{u}, E(\theta, \phi), P_{k}^{S}, \alpha_{k}^{S}, \tau_{S}, \tau_{D}\right)$, the resulting optimization problem with respect to $P_{k}^{D}$ is expressed as

$$
\begin{array}{ll}
\max _{P_{k}^{D}} & \lambda_{E E}^{\prime} \\
\text { s.t. } & R_{k}^{D} \geq R_{\min }^{D}, \forall k \in \mathcal{K}, \\
& \tau_{D}\left(\sum_{k=1}^{K} P_{k}^{D}+P_{C}^{D}\right) \leq E_{k}^{S}, \forall k \in \mathcal{K} .
\end{array}
$$


Problem (37) is challenging to tackle due to the non-convex function (37a) and constraint (37b). To tackle this problem, we employ the successive convex optimization technique. In particular, we first rewrite $R_{k}^{D}$ as a difference of two concave functions with respect to the power allocation, which is given by

$$
R_{k}^{D}=\tilde{R}_{k}^{D}-\hat{R}_{k}^{D}
$$

where

$$
\begin{aligned}
& \tilde{R}_{k}^{D}=\log _{2}\left(\sum_{i=1}^{K} g_{i, k}^{D} P_{i}^{D}+\sigma^{2}\right), \\
& \hat{R}_{k}^{D}=\log _{2}\left(\sum_{i \neq k}^{K} g_{i, k}^{D} P_{i}^{D}+\sigma^{2}\right) .
\end{aligned}
$$

Then, we let $P^{D r}$ be the $r$ th iteration of $P^{D}$. Using the first order Taylor expansion, the upper bound of (40) is rewritten as

$$
\begin{aligned}
\hat{R}_{k}^{D} & =\log _{2}\left(\sum_{i \neq k}^{K} g_{i, k}^{D} P_{i}^{D}+\sigma^{2}\right) \\
& \leq \sum_{i \neq k}^{K} C_{i, k}^{r}\left(P_{i}^{D}-P_{i}^{D r}\right)+\log _{2}\left(\sum_{i \neq k}^{K} g_{i, k}^{D} P_{i}^{D r}+\sigma^{2}\right) \\
& \triangleq \hat{R}_{k}^{D u b}
\end{aligned}
$$

where

$$
C_{i, k}^{r}=\frac{g_{i, k}^{D} \log _{2}(e)}{\sum_{l \neq k}^{K} g_{l, k}^{D} P_{l}^{D r}+\sigma^{2}} .
$$

By substituting (41) into problem (37), problem (37) is represented as

$$
\begin{array}{ll}
\max _{P_{k}^{D}} & \tau_{S} \sum_{k=1}^{K} R_{k}^{S}+\tau_{D} \sum_{k=1}^{K}\left(\tilde{R}_{k}^{D}-\hat{R}_{k}^{D u b}\right)-q E_{\text {total }} \\
\text { s.t. } & \log _{2}\left(\sum_{i=1}^{K} g_{i, k}^{D} P_{i}^{D}+\sigma^{2}\right)-\hat{R}_{k}^{D u b} \geq R_{m i n}^{D} \\
& \tau_{D}\left(\sum_{k=1}^{K} P_{k}^{D}+P_{C}^{D}\right) \leq E_{k}^{S}, \forall k \in \mathcal{K} .
\end{array}
$$

Thus, problem (43) is convex, and can be tackled by the standard convex optimization methods [33].

\section{E. Time Scheduling}

After solving the $\left(q, z_{u}, E(\theta, \phi), P_{k}^{S}, \alpha_{k}^{S}, P_{k}^{D}\right)$, problmen (13) is simplified as

$$
\begin{array}{cl}
\max _{\tau_{S}, \tau_{D}} & a_{0} \tau_{S}+a_{1} \tau_{D} \\
\text { s.t. } & a_{2} \tau_{S} \leq a_{3} \tau_{D} \\
& \tau_{S}+\tau_{D} \leq 1 \\
& 0 \leq \tau_{S}, \tau_{D} \leq 1,
\end{array}
$$

TABLE I

THE JOINT UAV LOCATION AND RESOURCE ALLOCATION ALGORITHM

1: Initialize $\mathbf{Z}^{n}, \mathbf{E}^{n}, \mathbf{P}_{\mathbf{S}}^{n}, \mathbf{A}_{\mathbf{S}}^{n}, \mathbf{P}_{\mathbf{D}}^{n}, \mathbf{T}^{n}$.

Calculate $\mathbf{Q}^{n}=\lambda_{E E}^{n}$, and set iterate index $\mathrm{n}=1$;

2: ITERATE

1) For given $\mathbf{Q}^{n}, \mathbf{E}^{n}, \mathbf{P}_{\mathbf{S}}^{n}, \mathbf{A}_{\mathbf{S}}^{n}, \mathbf{P}_{\mathbf{D}}^{n}, \mathbf{T}^{n}$,

solve problem (30) using the successive convex optimization technique, and obtain the optimal $\mathbf{Z}^{n+1}$. 2) For given $\mathbf{Q}^{n}, \mathbf{Z}^{n+1}, \mathbf{P}_{\mathbf{S}}^{n}, \mathbf{A}_{\mathbf{S}}^{n}, \mathbf{P}_{\mathbf{D}}^{n}, \mathbf{T}^{n}$, solve problem (34) according to the MOEA/D-based algorithm, and obtain the optimal $\mathbf{E}^{n+1}$.

3) With the given $\mathbf{Q}^{n}, \mathbf{Z}^{n+1}, \mathbf{E}^{n+1}, \mathbf{P}_{\mathbf{D}}^{n}, \mathbf{T}^{n}$, solve problem (35) according to Proposition 2, and obtain the optimal $\mathbf{P}_{\mathbf{S}}^{n+1}$ and $\mathbf{A}_{\mathbf{S}}^{n+1}$.

4) For given $\mathbf{Q}^{n}, \mathbf{Z}^{n+1}, \mathbf{E}^{n+1}, \mathbf{P}_{\mathbf{S}}^{n+1}, \mathbf{A}_{\mathbf{S}}^{n+1}, \mathbf{T}^{n}$, solve problem (43) by applying the successive convex optimization technique, and obtain the optimal $\mathbf{P}_{\mathbf{D}}^{n+1}$. 5) With the given $\mathbf{Q}^{n}, \mathbf{Z}^{n+1}, \mathbf{E}^{n+1}, \mathbf{P}_{\mathbf{S}}^{n+1}, \mathbf{A}_{\mathbf{S}}^{n+1}, \mathbf{P}_{\mathbf{D}}^{n+1}$, solve problem (44) and obtain the optimal $\mathbf{T}^{n+1}$. Calculate $\mathbf{Q}^{n+1}=\lambda_{E E}^{n+1}$, Update $\mathrm{n}=\mathrm{n}+1$.

3: UNTIL convergence.

where

$$
\begin{aligned}
a_{0} & =\sum_{k=1}^{K} R_{k}^{S}-q\left(P_{h o v}+\xi \sum_{k=1}^{K} P_{k}^{S}+P_{C}^{S}\right. \\
& \left.-\sum_{k=1}^{K} \eta\left(1-\alpha_{k}^{S}\right) g_{k}^{S} \sum_{i=1}^{K} P_{i}^{S}\right), \\
a_{1} & =\sum_{k=1}^{K} R_{k}^{D}-q\left(\sum_{k=1}^{K} P_{k}^{D}+P_{C}^{D}\right) \\
a_{2}= & \sum_{k=1}^{K} P_{k}^{D}+P_{C}^{D}, \\
a_{3}= & \left(1-\alpha_{k}^{S}\right) \eta g_{k} \sum_{i=1}^{K} P_{i}^{S} .
\end{aligned}
$$

Since problem (44) is linear with respect to the time allocation, it can be tackled using similar approaches.

Based on the previous subsections, the complete iterative algorithm for problem (13) is summarized in TABLE I. To simplify the description, let $\mathbf{Z}=\left\{z_{u}\right\}, \mathbf{E}=\{E(\theta, \phi)\}$, $\mathbf{P}_{\mathbf{S}}=\left\{P_{k}^{S}, \forall k\right\}, \mathbf{A}_{\mathbf{S}}=\left\{\alpha_{k}^{S}, \forall k\right\}, \quad \mathbf{P}_{\mathbf{D}}=\left\{P_{k}^{D}, \forall k\right\}, \mathbf{T}=$ $\left\{\tau_{S}, \tau_{D}\right\}, \mathbf{Q}=\left\{q^{*}\right\}$. In each iteration, EE is maximized over $\mathbf{Z}$, while keeping $\left(\mathbf{Q}, \mathbf{E}, \mathbf{P}_{\mathbf{S}}, \mathbf{A}_{\mathbf{S}}, \mathbf{P}_{\mathbf{D}}, \mathbf{T}\right)$ 's fixed. For a given $\left(\mathbf{Q}, \mathbf{Z}, \mathbf{P}_{\mathbf{S}}, \mathbf{A}_{\mathbf{S}}, \mathbf{P}_{\mathbf{D}}, \mathbf{T}\right)$, the set of $\mathbf{E}$ is obtained via solving problem (34). Then, we fix the $\left(\mathbf{Q}, \mathbf{Z}, \mathbf{E}, \mathbf{P}_{\mathbf{D}}, \mathbf{T}\right)$ to calculate $\left(\mathbf{P}_{\mathbf{S}}, \mathbf{A}_{\mathbf{S}}\right)$ via solving problem (35) by the standard convex optimization methods. In addition, with the fixed $\left(\mathbf{Q}, \mathbf{Z}, \mathbf{E}, \mathbf{P}_{\mathbf{S}}, \mathbf{A}_{\mathbf{S}}, \mathbf{T}\right), \mathbf{P}_{\mathbf{D}}$ can be obtained by solving problem (43). Finally, we can obtain $\mathbf{T}$ via tackling problem (44) and calculating $\mathbf{Q}$ based on (17). The algorithm terminates until convergence is reached. 


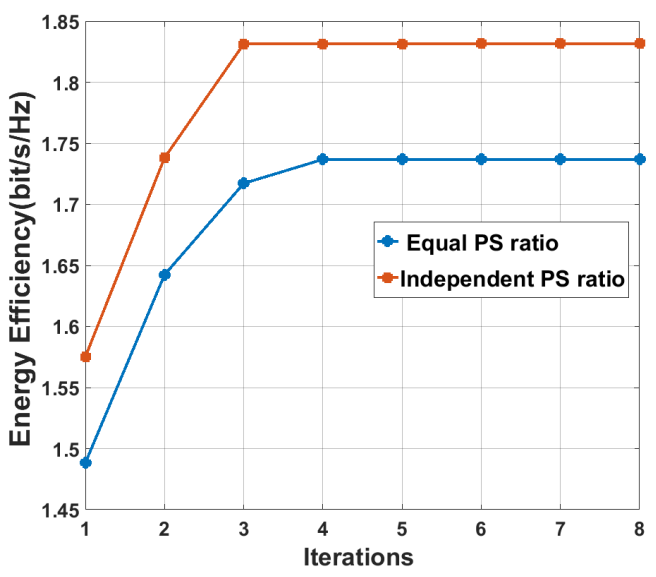

Fig. 2. The convergence behavior of the proposed joint UAV location and resource allocation algorithm with different PS ratio schemes.

\section{NUMERICAL RESULTS}

In this section, the simulation results are presented in order to verify the superiority of the proposed algorithm. In our simulations, the reference channel power gain at $d_{0}=1 \mathrm{~m}$ is assumed to be $\rho_{0}=-30 \mathrm{~dB}$. The UAV is mounted with an 8 $\times 8$ antenna array which is partitioned into several sub-arrays according to the number of D2D pairs. The maximum transmit power of UAV is set as $P_{\max }=20 \mathrm{~W}$, and UAV is assumed to fly at a fixed altitude $H=10 \mathrm{~m}$ with the hover power $P_{\text {hov }}=110 \mathrm{~W}$ [34]. In addition, the static circuit power at the D2D-TX is set as $P_{C}^{S}=5 \mathrm{~mW}$, and is set as $P_{C}^{D}=$ $10 \mu \mathrm{W}$ at the D2D-RX. The EH efficiency $\eta$ is set to 0.1 , and the reciprocal of the power amplifier drain efficiency $\zeta$ is set to 0.38 . In order to satisfy the QoS requirements for all the D2D-pairs, the minimum transmission rate constraint of SWIPT phase and D2D phase is set to $2 \mathrm{bit} / \mathrm{s} / \mathrm{Hz}$ and $1 \mathrm{bit} / \mathrm{s} / \mathrm{Hz}$ respectively.

In the first simulation, we study the convergence behavior of our proposed algorithm with different PS ratio strategies. Specifically, we consider both the equal PS ratio case and the independent PS ratio case with $K=4$ D2D pairs. The PS ratio of different D2D pairs are set as the same value in the first case whilst the PS radio is optimized based on the NOMA scheme in the other case. As is shown in Fig. 2, the EE of the both two cases converge to a fixed value within three iterations. In addition, the proposed joint UAV location and resource allocation algorithm with independent PS ratio can achieve higher EE, but it also cost higher computational complexity.

In the next simulation, we investigate the $\mathrm{EE}$ of the considered D2D communications underlaying UAV-assisted IIoT system versus the PS ratio. The number of D2D pairs is set to $K=4$ with equal PS ratio scheme. As shown in Fig. 3, the relationship between the EE and the PS ratio is quasiconcave. This demonstrates that there is a trade-off between the PS scheme for EH and ID. In particular, a high PS ratio reduces the energy harvested by D2D-TXs, which in turn reduces the throughput in D2D phase. In contrast, a low PS ratio may increase the energy harvested by D2D-TXs. In

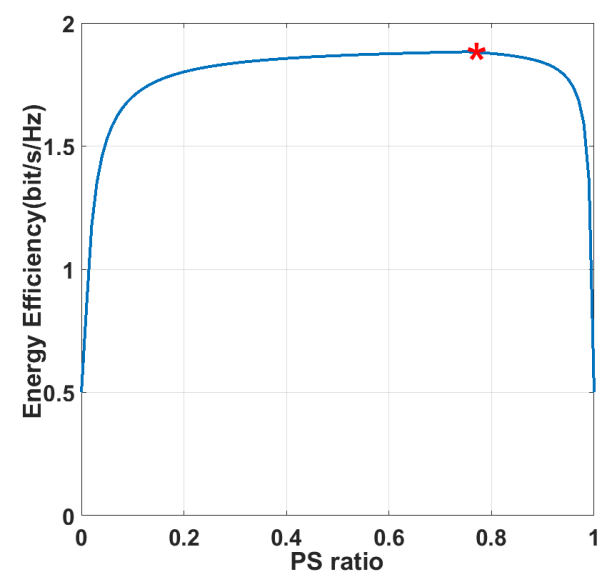

Fig. 3. The energy efficiency of the D2D communications underlaying UAVassisted IIoT system versus the PS ratio.

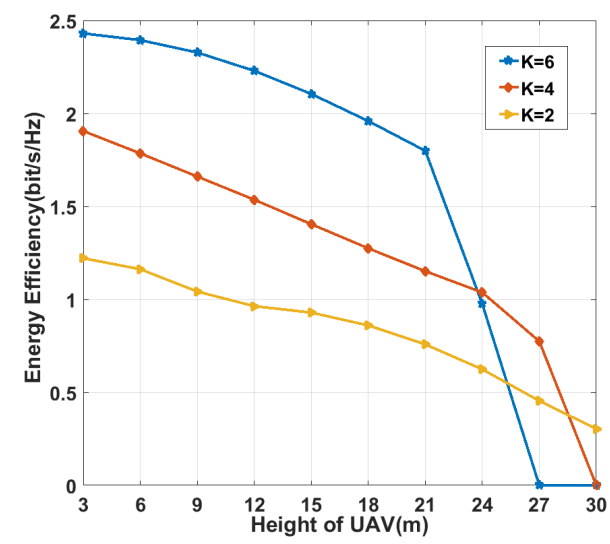

Fig. 4. The energy efficiency of the D2D communications underlaying UAVassisted IIoT system versus the height of UAV.

order to satisfy the minimum transmission rate constraints in the SWIPT phase, the UAV has to use a larger transmission power, resulting in a decrease in the EE performance. In other words, a suitable value of PS ratio can achieve a significant improvement in EE performance.

Then, the EE performance of the proposed algorithm under various constraints are presented in Fig. 4, Fig. 5, Fig. 6 and Fig. 7. The number of D2D pairs is set to 2,4 and 6 respectively. Firstly, the EE of the D2D communications underlaying UAV-assisted IIoT system versus the height of UAV is evaluated under different number of D2D pairs. In particular, we set the height of UAV within the range of $3 \mathrm{~m}$ to $30 \mathrm{~m}$. As it can be shown in Fig. 4, the EE achieved by our proposed algorithm decreases monotonically with the height of UAV. Furthermore, in the case of $K=6$ D2D pairs, the EE decreases rapidly when the height of UAV reaches at $21 \mathrm{~m}$. This is due to the fact that increasing the UAV's height will increase the path loss, and hence restricts the improvement of the EE performance. In addition, the EE is non-decreasing with the number of D2D pairs. This is due to the fact that a larger number of D2D pairs is capable of enhancing the diversity gain. Therefore, a suitable number of D2D pairs can 


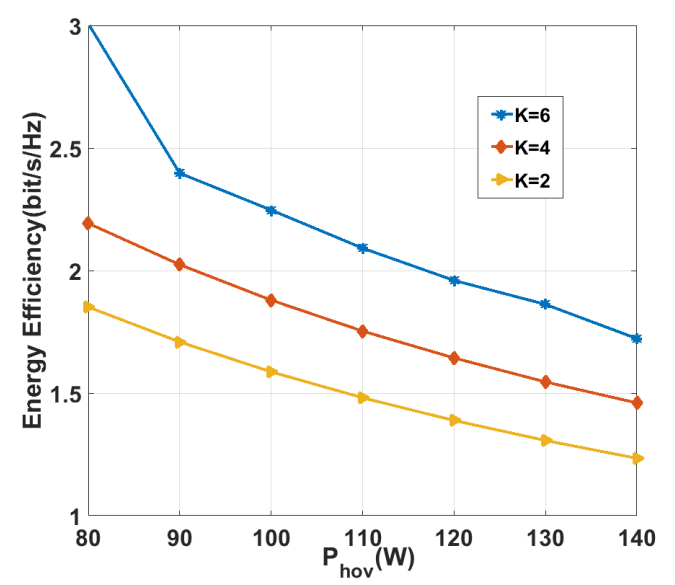

Fig. 5. The energy efficiency of the D2D communications underlaying UAVassisted IIoT system versus the hover power of UAV.

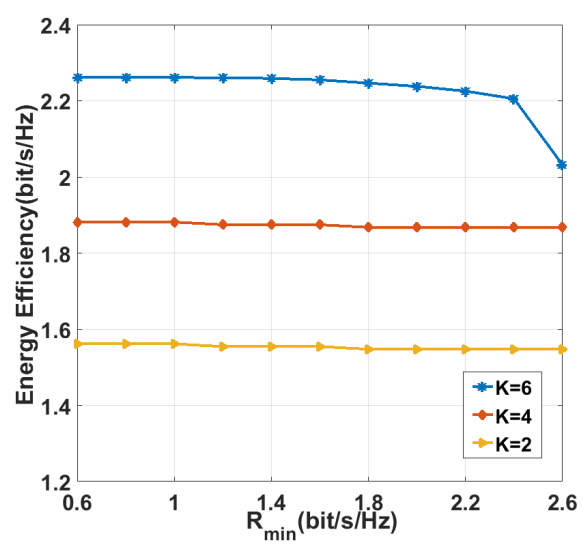

Fig. 6. The energy efficiency of the D2D communications underlaying UAVassisted IIoT system versus minimum rate constraint.

achieve a better EE performance.

We next investigate the $\mathrm{EE}$ of the D2D communications underlaying UAV-assisted IIoT system versus the hover power of UAV under different number of D2D pairs. The hover power of UAV is set within the range of $80 \mathrm{~W}$ to $140 \mathrm{~W}$. As shown in Fig. 5, the EE achieved by our proposed method is monotonically non-increasing with the increasing of the hover power of UAV. This is because that increasing the hover power of UAV will increase the total energy consumption of the system, and thus restrict the improvement of the EE. Similarly, the proposed joint UAV location and resource allocation algorithm with large number of D2D pairs achieves a higher EE performance.

In the next simulations, we study the EE of the D2D communications underlaying UAV-assisted IIoT system versus minimum rate constraint under different number of D2D pairs. The minimum rate constraints in SWIPT phase and D2D phase set as the same value within the ranger of $0.6 \mathrm{bit} / \mathrm{s} / \mathrm{Hz}$ to $2.6 \mathrm{bit} / \mathrm{s} / \mathrm{Hz}$. As it can be seen in Fig.6, the EE is the same under a certain minimum rate constraint $R_{\min }, 0.6 \leq R_{\min } \leq$ $2.4 \mathrm{bit} / \mathrm{s} / \mathrm{Hz}$, but drops after when $K=6$. The reason is that the proposed joint UAV location and resource allocation algo-

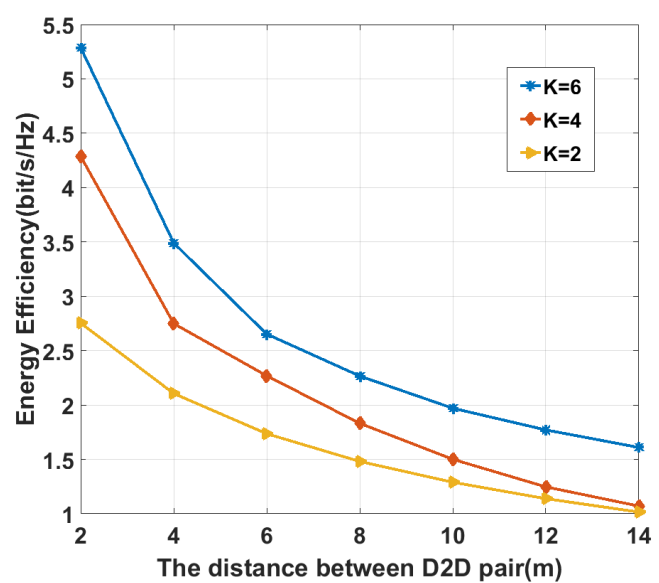

Fig. 7. The energy efficiency of IIoT devices versus the distance between each D2D pair.

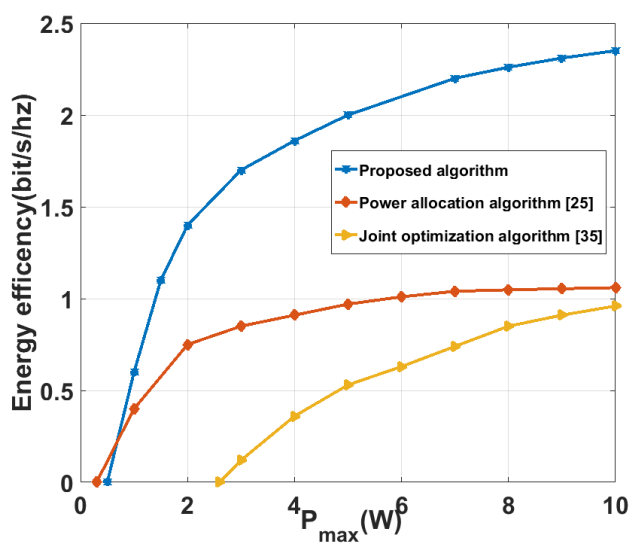

Fig. 8. The energy efficiency of all IIoT devices versus the power budget under different resource allocation schemes.

rithm can guarantee the QoS requirements sufficiently when the minimum transmission rate constraints are sufficiently small. However, when $R_{\min } \geq 2.4 \mathrm{bit} / \mathrm{s} / \mathrm{Hz}$, the EE decreases in the case of $K=6 \mathrm{D} 2 \mathrm{D}$ pairs. This is because more transmit power is needed to satisfy the increasing minimum rate constrains ,resulting in a reduction of energy harvested by D2D-TX, and thus limits the improvement of the system throughput. This results in a decrease in EE performance.

We then study the EE of the D2D communications underlaying UAV-assisted IIoT system versus the distance between each D2D pair. In particular, we set the distance between each D2D pair within the range of $2 \mathrm{~m}$ to $14 \mathrm{~m}$. As shown in Fig.7, the EE is non-increasing with the distance between each D2D pair. In particular, when the distance of D2D pairs is small, the interference received by D2D-RX from other D2D pairs is sufficiently low compared to the signal received by its D2DTX, thus the achievable EE is high. In contrast, as the distance of D2D pairs increases, the power consumption become larger and the achievable rate become lower, which decrease the EE.

In the last simulation, we study the EE of the D2D communications underlaying UAV-assisted IIoT system versus the power budget under different resource allocation schemes. To 
show the EE performance of our proposed algorithm, we compare with the "power control algorithm" in the UAVassisted D2D networks [25] and the "joint optimization" algorithm in the UAV-enabled IoT networks [35]. We assume that there are $K=4 \mathrm{D} 2 \mathrm{D}$ pairs and the minimum rate constraints is $1 \mathrm{bit} / \mathrm{s} / \mathrm{Hz}$. As it can be seen in Fig. 8, the EE obtained by our proposed algorithm outperforms both the other two algorithms. This is due to the fact that we apply the beamforming technique to improve the channel power gain, and therefore enhances the system performance. In particular, our proposed algorithm enables the UAV-mounted antenna array to form the directional beams which can compensate the high propagation loss. Furthermore, our algorithm adopts NOMA to enable the UAV to communicate with multiple D2D-TXs simultaneously, which further increases the EE of the network.

\section{CONClusion}

In this paper, we propose a joint UAV location and resource allocation algorithm for the D2D communications underlaying UAV-assisted industrial IoT network. In particular, in the SWIPT phase, the UAV serves as a flying BS to transmit energy and information to D2D-TXs. Then in the D2D phase, D2D-TXs transmit information to D2D-RXs using the harvested energy. Our aim is to maximize the EE of the network while satisfying the constraints of minimum transmission rate and the power budget. The formulated EE maximization problem involves joint optimization of the UAV location, beam pattern design, power allocation and time scheduling, which is non-convex and challenging to solve. To solve this problem, by applying the Dinkelbach method, the successive convex optimization techniques and the MOEA/D algorithm, we propose a iterative resource allocation algorithm to optimize the variables sequentially. Numerical results illustrate that the EE obtained by the proposed algorithm outperform the existing works.

\section{APPENDIX}

Firstly, we prove that the objective function (35a) is concave in the transmit power of the UAV. To simplify the description, we let $\beta_{k}=\sum_{i=k}^{K} P_{i}^{S}, \alpha^{S}=\alpha, g^{S}=g$. The objective function can be reformulated as

$$
\begin{aligned}
& \Lambda_{E E}(\boldsymbol{P}) \\
& =\sum_{k=1}^{K} \log _{2}\left(\frac{\sigma^{2}+\alpha_{k} g_{k} \beta_{k}}{\sigma^{2}+\alpha_{k} g_{k} \beta_{k+1}}\right) \\
& -q\left(\zeta \beta_{1}+P_{C}^{S}-\eta \sum_{k=1}^{K}\left(1-\alpha_{k}\right) g_{k} \beta_{1}\right) \\
& =\sum_{k=2}^{K}\left(\log _{2}\left(\sigma^{2}+\alpha_{k} g_{k} \beta_{k}\right)-\log _{2}\left(\sigma^{2}+\alpha_{k-1} g_{k-1} \beta_{k}\right)\right) \\
& -q\left(\zeta \beta_{1}+P_{C}-\eta \sum_{k=1}^{K}\left(1-\alpha_{k}\right) g_{k} \beta_{1}\right) \\
& -\log _{2}\left(\sigma^{2}\right)+\log _{2}\left(\sigma^{2}+\alpha_{1} g_{1} \beta_{1}\right) .
\end{aligned}
$$

Then the first-order derivative of $\Lambda_{E E}(\boldsymbol{P})$ can be expressed as

$$
\begin{aligned}
& \frac{\partial \Lambda_{E E}(\boldsymbol{P})}{\partial P_{m}} \\
& =\frac{1}{\ln 2} \cdot\left(\frac{\alpha_{1} g_{1}}{\sigma^{2}+\alpha_{1} g_{1} \beta_{1}}+\sum_{k=2}^{m}\left(\frac{\alpha_{k} g_{k}}{\sigma^{2}+\alpha_{k} g_{k} \beta_{k}}\right.\right. \\
& \left.\left.-\frac{\alpha_{k-1} g_{k-1}}{\sigma^{2}+\alpha_{k-1} g_{k-1} \beta_{k}}\right)\right)-q\left(\zeta-\eta \sum_{k=1}^{K}\left(1-\alpha_{k}\right) g_{k}\right) .
\end{aligned}
$$

Furthermore, the second-order derivative of $\Lambda_{E E}(\boldsymbol{P})$ is denoted as

$$
\begin{aligned}
& \frac{\partial^{2} \Lambda_{E E}(\boldsymbol{P})}{\partial P_{m} \partial P_{l}} \\
& =-\frac{1}{\ln 2} \cdot \sum_{k=2}^{j}\left(\frac{\alpha_{k}^{2} g_{k}^{2}}{\left(\sigma^{2}+\alpha_{k} g_{k} \beta_{k}\right)^{2}}-\frac{\alpha_{k-1}^{2} g_{k-1}^{2}}{\left(\sigma^{2}+\alpha_{k-1} g_{k-1} \beta_{k}\right)^{2}}\right) \\
& -\frac{1}{\ln 2} \cdot \frac{\alpha_{1}^{2} g_{1}^{2}}{\left(\sigma^{2}+\alpha_{1} g_{1} \beta_{1}\right)^{2}},
\end{aligned}
$$

Let $H_{m}=\frac{\partial^{2} \Lambda_{E E}(\boldsymbol{P})}{\partial P_{m}^{2}}$. According to (48), it is obvious that $\frac{\partial^{2} \Lambda_{E E}(\boldsymbol{P})}{\partial P_{m} \partial P_{l}}=H_{m}$ when $l \geq m$, and $\frac{\partial^{2} \Lambda_{E E}(\boldsymbol{P})}{\partial P_{m} \partial P_{l}}=H_{l}$ when $l \leq m$. Then, the Hessian matrix can be expressed as

$$
\mathbf{H}=\left(\begin{array}{cccc}
H_{1} & H_{1} & \cdots & H_{1} \\
H_{1} & H_{2} & \cdots & H_{2} \\
\vdots & \vdots & & \vdots \\
H_{1} & H_{2} & \cdots & H_{K}
\end{array}\right) .
$$

Then, we define the matrix $\mathbf{Q}=-\mathbf{H}$, in which the $k$-th order principal minor can be formulated as

$$
\begin{aligned}
Q_{k} & =\left|\begin{array}{cccc}
-H_{1} & -H_{1} & \cdots & -H_{1} \\
-H_{1} & -H_{2} & \cdots & -H_{2} \\
\vdots & \vdots & & \vdots \\
-H_{1} & -H_{2} & \cdots & -H_{k}
\end{array}\right| \\
& =\left|\begin{array}{cccc}
-H_{1} & -H_{1} & \cdots & -H_{1} \\
0 & H_{1}-H_{2} & \cdots & H_{1}-H_{2} \\
\vdots & \vdots & & \vdots \\
0 & 0 & \cdots & H_{k-1}-H_{k}
\end{array}\right| \\
& =\left\{\begin{array}{c}
-H_{1}, k=1 \\
-H_{1} \prod_{i=2}^{k}\left(H_{i-1}-H_{i}\right), 2 \leq k \leq K .
\end{array}\right.
\end{aligned}
$$

Since $-H_{1}=-\frac{1}{\ln 2} \cdot \frac{\alpha_{1}^{2} g_{1}^{2}}{\left(\sigma^{2}+\alpha_{1} g_{1} \beta_{1}\right)^{2}} \geq 0$, and for $2 \leq i \leq K$,

$$
\begin{aligned}
H_{i-1}-H_{i} & =\frac{1}{\ln 2} \cdot\left(\frac{\alpha_{i}^{2} g_{i}^{2}}{\left(\sigma^{2}+g_{i} \beta_{i}\right)^{2}}-\frac{\alpha_{i-1}^{2} g_{i-1}^{2}}{\left(\sigma^{2}+g_{i-1} \beta_{i}\right)^{2}}\right) \\
& =\frac{1}{\ln 2} \cdot\left(\frac{1}{\left(\frac{\sigma^{2}}{\alpha_{i} g_{i}}+\beta_{i}\right)^{2}}-\frac{1}{\left(\frac{\sigma^{2}}{\alpha_{i-1} g_{i-1}}+\beta_{i}\right)^{2}}\right) \\
& >0 .
\end{aligned}
$$

We have proved that $Q_{k} \geq 0$, and thus $\mathbf{Q}=-\mathbf{H} \succeq \mathbf{0}$ and $\mathbf{H} \preceq \mathbf{0}$. Therefore, the objective function is concave in $\boldsymbol{P}$. 
Next, we prove that the objective function (35a) is concave in the PS ratio. The first-order derivative of $\Lambda_{E E}(\boldsymbol{\alpha})$ is given by

$$
\begin{aligned}
& \frac{\partial \Lambda_{E E}(\boldsymbol{\alpha})}{\partial \alpha_{m}} \\
& =\frac{1}{\ln 2} \cdot\left(\frac{\beta_{1} g_{1}}{\sigma^{2}+\alpha_{1} g_{1} \beta_{1}}+\sum_{k=2}^{m}\left(\frac{\beta_{k} g_{k}}{\sigma^{2}+\alpha_{k} g_{k} \beta_{k}}\right.\right. \\
& \left.\left.-\frac{\beta_{k-1} g_{k-1}}{\sigma^{2}+\alpha_{k-1} g_{k-1} \beta_{k}}\right)\right)-q\left(\zeta-\eta \sum_{k=1}^{K}\left(1-\alpha_{k}\right) g_{k}\right) .
\end{aligned}
$$

The second-order derivative of $\Lambda_{E E}(\boldsymbol{\alpha})$ can be denoted as

$$
\begin{aligned}
& \frac{\partial^{2} \Lambda_{E E}(\boldsymbol{\alpha})}{\partial \alpha_{m} \partial \alpha_{l}} \\
& =-\frac{1}{\ln 2} \cdot \sum_{k=2}^{j}\left(\frac{\beta_{k}^{2} g_{k}^{2}}{\left(\sigma^{2}+\alpha_{k} g_{k} \beta_{k}\right)^{2}}-\frac{\beta_{k-1}^{2} g_{k-1}^{2}}{\left(\sigma^{2}+\alpha_{k-1} g_{k-1} \beta_{k}\right)^{2}}\right) \\
& -\frac{1}{\ln 2} \cdot \frac{\beta_{1}^{2} g_{1}^{2}}{\left(\sigma^{2}+\alpha_{1} g_{1} \beta_{1}\right)^{2}} .
\end{aligned}
$$

Let $H_{m}=\frac{\partial^{2} \Lambda_{E E}(\boldsymbol{\alpha})}{\partial \alpha_{m}^{2}}$, according to (53), the Hessian matrix is expressed as

$$
\mathbf{H}=\left(\begin{array}{cccc}
H_{1} & H_{1} & \cdots & H_{1} \\
H_{1} & H_{2} & \cdots & H_{2} \\
\vdots & \vdots & & \vdots \\
H_{1} & H_{2} & \cdots & H_{K}
\end{array}\right) .
$$

Then, we define the matrix $\mathbf{Q}=-\mathbf{H}$, and the $k$-th order principal minor can be formulated as

$$
\begin{aligned}
Q_{k} & =\left|\begin{array}{cccc}
-H_{1} & -H_{1} & \cdots & -H_{1} \\
-H_{1} & -H_{2} & \cdots & -H_{2} \\
\vdots & \vdots & & \vdots \\
-H_{1} & -H_{2} & \cdots & -H_{k}
\end{array}\right| \\
& =\left|\begin{array}{cccc}
-H_{1} & -H_{1} & \cdots & -H_{1} \\
0 & H_{1}-H_{2} & \cdots & H_{1}-H_{2} \\
\vdots & \vdots & & \vdots \\
0 & 0 & \cdots & H_{k-1}-H_{k}
\end{array}\right| \\
& =\left\{\begin{array}{l}
-H_{1}, k=1 \\
-H_{1} \prod_{i=2}^{k}\left(H_{i-1}-H_{i}\right), 2 \leq k \leq K .
\end{array}\right.
\end{aligned}
$$

Note that $-H_{1}=-\frac{1}{\ln 2} \cdot \frac{\beta_{1}^{2} g_{1}^{2}}{\left(\sigma^{2}+\alpha_{1} g_{1} \beta_{1}\right)^{2}} \geq 0$, and for $2 \leq i \leq K$,

$$
\begin{aligned}
H_{i-1}-H_{i} & =\frac{1}{\ln 2} \cdot\left(\frac{g_{i}^{2} \beta_{i}^{2}}{\left(\sigma^{2}+g_{i} \beta_{i}\right)^{2}}-\frac{g_{i-1}^{2} \beta_{i}^{2}}{\left(\sigma^{2}+g_{i-1} \beta_{i}\right)^{2}}\right) \\
& =\frac{1}{\ln 2} \cdot\left(\frac{\beta_{i}^{2}}{\left(\frac{\sigma^{2}}{g_{i}}+\beta_{i}\right)^{2}}-\frac{\beta_{i}^{2}}{\left(\frac{\sigma^{2}}{g_{i-1}}+\beta_{i}\right)^{2}}\right)
\end{aligned}
$$$$
>0
$$

which implies that $\mathbf{Q}=-\mathbf{H} \succeq \mathbf{0}$ and $\mathbf{H} \preceq \mathbf{0}$, we can conclude that the objective function is concave in $\alpha$.

\section{REFERENCES}

[1] S. Li, L. Da Xu, and S. Zhao, "5G internet of things: A survey," J. Ind. Inf. Integr., vol. 10, pp. 1-9, Jun. 2018.

[2] G. A. Akpakwu, B. J. Silva, G. P. Hancke, and A. M. Abu-Mahfouz, "A survey on 5G networks for the internet of things: Communication technologies and challenges," IEEE Access, vol. 6, pp. 3619-3647, Dec. 2017.

[3] L. R. Varshney, "Transporting information and energy simultaneously," in IEEE Int. Symp. Info. Theory. IEEE, Jul. 2008, pp. 1612-1616.

[4] J. Huang, C.-C. Xing, and M. Guizani, "Power allocation for D2D communications with SWIPT," IEEE Trans. Wireless Commun., vol. 19, no. 4, pp. 2308-2320, Jan. 2020.

[5] D. Feng, L. Lu, Y. Yuan-Wu, G. Y. Li, G. Feng, and S. Li, "Deviceto-device communications underlaying cellular networks," IEEE Trans. Commun., vol. 61, no. 8, pp. 3541-3551, Aug. 2013.

[6] T. D. Hoang, L. B. Le, and T. Le-Ngoc, "Joint mode selection and resource allocation for relay-based D2D communications," IEEE Commun. Lett., vol. 21, no. 2, pp. 398-401, Oct. 2016.

[7] D. Wu, J. Wang, R. Q. Hu, Y. Cai, and L. Zhou, "Energy-efficient resource sharing for mobile device-to-device multimedia communications," IEEE Trans. Veh. Technol., vol. 63, no. 5, pp. 2093-2103, Jun. 2014.

[8] T.-W. Ban and B. C. Jung, "On the link scheduling for cellular-aided device-to-device networks," IEEE Trans. Veh. Technol., vol. 65, no. 11, pp. 9404-9409, Nov. 2016.

[9] A. Al-Hourani, S. Kandeepan, and S. Lardner, "Optimal LAP altitude for maximum coverage," IEEE Wireless Commun. Lett., vol. 3, no. 6 , pp. 569-572, Jul. 2014.

[10] J. Lyu, Y. Zeng, R. Zhang, and T. J. Lim, "Placement optimization of UAV-mounted mobile base stations," IEEE Wireless Commun. Lett., vol. 21, no. 3, pp. 604-607, Nov. 2016.

[11] F. Jiang and A. L. Swindlehurst, "Optimization of UAV heading for the ground-to-air uplink," IEEE J. Sel. Areas Commun., vol. 30, no. 5, pp. 993-1005, Jun. 2012.

[12] J. Xu, Y. Zeng, and R. Zhang, "UAV-enabled wireless power transfer: Trajectory design and energy optimization," IEEE Trans. Wireless Commun., vol. 17, no. 8, pp. 5092-5106, May. 2018.

[13] W. Feng, N. Zhao, S. Ao, J. Tang, X. Zhang, Y. Fu, D. K. So, and K.$\mathrm{K}$. Wong, "Joint 3D trajectory and power optimization for UAV-aided mmWave MIMO-NOMA networks," IEEE Trans. Commun., Dec. 2020.

[14] X. Hong, P. Liu, F. Zhou, S. Guo, and Z. Chu, "Resource allocation for secure UAV-assisted SWIPT systems," IEEE Access, vol. 7, pp. 24248 24 257, Feb. 2019.

[15] Z. Yang, Z. Ding, P. Fan, and N. Al-Dhahir, "A general power allocation scheme to guarantee quality of service in downlink and uplink NOMA systems," IEEE Trans. Wireless Commun., vol. 15, no. 11, pp. 7244 7257, Aug. 2016.

[16] J. Tang, J. Luo, M. Liu, D. K. So, E. Alsusa, G. Chen, K.-K. Wong, and J. A. Chambers, "Energy efficiency optimization for NOMA with SWIPT," IEEE J. Sel. Topics Signal Process., vol. 13, no. 3, pp. 452466, Feb. 2019.

[17] A. Masaracchia, L. D. Nguyen, T. Q. Duong, C. Yin, O. A. Dobre, and E. Garcia-Palacios, "Energy-Efficient and throughput fair resource allocation for TS-NOMA UAV-Assisted communications," IEEE Trans. Commun., vol. 68, no. 11, pp. 7156-7169, Aug. 2020.

[18] J. Zhao, Y. Wang, Z. Fei, X. Wang, and Z. Miao, "NOMA-Aided UAV data collection system: Trajectory optimization and communication design," IEEE Access, vol. 8, pp. 155 843-155 858, Aug. 2020.

[19] W. Feng, J. Tang, N. Zhao, Y. Fu, X. Zhang, K. Cumanan, and K.-K. Wong, "NOMA-based UAV-aided networks for emergency communications," China Commun., vol. 17, no. 11, pp. 54-66, Nov. 2020.

[20] X. Liu, Q. Zhang, W. Chen, H. Feng, L. Chen, F. M. Ghannouchi, and Z. Feng, "Beam-oriented digital predistortion for 5G massive MIMO hybrid beamforming transmitters," IEEE Trans. Microw. Theory Techn., vol. 66, no. 7, pp. 3419-3432, May. 2018.

[21] W. Feng, N. Zhao, S. Ao, J. Tang, X. Zhang, Y. Fu, D. K. So, and K.-K. Wong, "Joint 3D trajectory design and time allocation for UAV-enabled wireless power transfer networks," IEEE Trans. Veh. Technol., vol. 69, no. 9, pp. 9265-9278, Feb. 2020.

[22] T. Liu, X. Qu, F. Yin, and Y. Chen, "Energy efficiency maximization for wirelessly powered sensor networks with energy beamforming," IEEE Commun. Lett., vol. 23, no. 12, pp. 2311-2315, Sep. 2019.

[23] S. He, Y. Huang, S. Jin, F. Yu, and L. Yang, "Max-min energy efficient beamforming for multicell multiuser joint transmission systems," IEEE Commun. Lett., vol. 17, no. 10, pp. 1956-1959, Aug. 2013. 
[24] H. T. Nguyen, H. D. Tuan, T. Q. Duong, H. V. Poor, and W.-J. Hwang, "Joint D2D assignment, bandwidth and power allocation in cognitive UAV-Enabled networks," IEEE Trans. Cogn. Commun. Netw., vol. 6, no. 3, pp. 1084-1095, Jan. 2020.

[25] H. Wang, J. Chen, G. Ding, and S. Wang, "D2D communications underlaying UAV-assisted access networks," IEEE Access, vol. 6, pp. 46244-46255, Aug. 2018.

[26] F. Huang, J. Chen, H. Wang, G. Ding, Z. Xue, Y. Yang, and F. Song, "UAV-assisted SWIPT in internet of things with power splitting: Trajectory design and power allocation," IEEE Access, vol. 7, pp. 68260 68270, May. 2019.

[27] Q. Zhang and H. Li, "MOEA/D: A multiobjective evolutionary algorithm based on decomposition," IEEE Trans. Evol. Comput., vol. 11, no. 6, pp. 712-731, Dec. 2007.

[28] N. Rupasinghe, Y. Yapıcı, I. Güvenç, and Y. Kakishima, "Nonorthogonal multiple access for mmwave drone networks with limited feedback," IEEE Trans. Commun., vol. 67, no. 1, pp. 762-777, Aug. 2018.

[29] L. Zhu, J. Zhang, Z. Xiao, X. Cao, D. O. Wu, and X.-G. Xia, "3-D beamforming for flexible coverage in millimeter-wave UAV communications," IEEE Wireless Commun. Lett., vol. 8, no. 3, pp. 837-840, Jun. 2019.

[30] Y. Chen, B. Ai, Y. Niu, R. He, Z. Zhong, and Z. Han, "Resource allocation for device-to-device communications in multi-cell multi-band heterogeneous cellular networks," IEEE Trans. Veh. Technol., vol. 68, no. 5, pp. 4760-4773, Mar. 2019.

[31] W. Dinkelbach, "On nonlinear fractional programming," Manage. Sci., vol. 13, no. 7, pp. 492-498, Mar. 1967.

[32] Y. Zeng, R. Zhang, and T. J. Lim, "Throughput maximization for UAV-enabled mobile relaying systems," IEEE Trans. Commun., vol. 64 , no. 12, pp. 4983-4996, Dec. 2016.

[33] S. Boyd, S. P. Boyd, and L. Vandenberghe, Convex optimization. Cambridge university press, 2004.

[34] C. Di Franco and G. Buttazzo, "Energy-aware coverage path planning of uavs," in IEEE Int. Conf. Auton. Robot Syst. Competitions. IEEE, Apr. 2015, pp. 111-117.

[35] Y. Liu, K. Liu, J. Han, L. Zhu, Z. Xiao, and X.-G. Xia, "Resource allocation and 3D placement for UAV-Enabled Energy-Efficient IoT communications," IEEE Internet Things J., Jun. 2020. 